\title{
On the nature of the shape coexistence and the quantum phase transition phenomena: lead region and $\mathrm{Zr}$ isotopes
}

\author{
José-Enrique García-Ramos ${ }^{1,2, \star}$ and Kris Heyde ${ }^{3, \star \star}$ \\ ${ }^{1}$ Departamento de Ciencias Integradas y CEAFMC Universidad de Huelva, 21071 Huelva \\ ${ }^{2}$ Instituto Carlos I de Física Teórica y Computacional, Universidad de Granada, Fuentenueva s/n, 18071 Granada, Spain \\ ${ }^{3}$ Department of Physics and Astronomy, Ghent University, Proeftuinstraat, 86 B-9000 Gent, Belgium
}

\begin{abstract}
The goal of this contribution is to analyze the connection between shape coexistence and quantum phase transition, two seemingly unrelated phenomena that share common aspects, namely, the rapid change in the ground state structure along an isotope chain or the presence of several minima at the mean-field level. To illustrate the similarities and differences between both phenomena, we will focus in the $\mathrm{Pb}$ region, in particular in $\mathrm{Pt}$ and $\mathrm{Hg}$ isotopes, as well as in $\mathrm{Zr}$ isotopes.
\end{abstract}

\section{Introduction}

The atomic nuclei can present different shapes, namely, spherical, axially deformed, either prolate or oblate, or even triaxial. In general, the shape evolves in a gradual way as one passes from a nucleus to its neighbor, but in certain cases the change can be very abrupt. This is the key element for the appearance of a quantum phase transition (QPT) [1]. On the other hand, in a given nucleus different shapes can coexist in the energy spectrum, leading to the appearance of shape coexistence [2]. The presence of extra configurations can be easily singled out in the very conspicuous parabolic behaviour in the energy systematics centered around the mid-shell. Moreover, another relevant indicator of the presence of intruder configurations is the lowering of the first excited $0^{+}$state that presents a minimum also around the mid-shell.

Along this contribution we try to disentangle which are the relationships and differences between both phenomena. To this end, we analyze three different chains of nuclei, $\mathrm{Pt}, \mathrm{Hg}$, and $\mathrm{Zr}$ which are ideal cases to see the competition between QPT and shape coexistence phenomena.

\section{Shape coexistence and quantum phase transition in a nutshell}

Shape coexistence in nuclear physics was first proposed by Morinaga in the 1950's and since them it has given rise to a property of atomic nuclei that appears throughout the entire nuclear landscape, especially to those nuclei at or near shell or sub-shell closures [2]. Shape coexistence presents certain distinct experimental features: a $\mathrm{U}$ shape in the energy systematics of certain excited bands,

\footnotetext{
^e-mail: enrique.ramos@dfaie.uhu.es

$\star \star$ e-mail: kris.heyde@ugent.be
}

lowering of certain excited $0^{+}$states, a rapid change in the value of spectroscopic quadrupole moments, and the existence of strong E0 transitions. All of them are enhanced and present an almost symmetric behaviour with respect to the corresponding mid-shell. The appearance of intruder configurations reflects the competition between the energy gap, that tends to maintain spherical shapes, and the residual interaction, that favours the deformation of the nucleus and lower, in some cases considerably, the excitation energy of the intruder states [2].

Shape coexistence can be understood in terms of two major theoretical approaches, namely the spherical shell model and the mean field. According to the shell model approach, the nucleus is described accordingly as an inert core and a set of valence nucleons that occupy certain orbits and interact amongst them through a residual two-body interaction. The promotion of pairs of nucleons across the shell gaps at shell and subshell closures is the mechanism responsible of the creation of intruder configurations. From the point of view of mean-field theory, using the self-consistent Hartree-Fock-Bogoliubov (HFB) theory, one obtains an energy surface depending on certain deformation parameters. In this framework, the ground band will correspond to the deepest minimum, but the others can be interpreted as intruder configurations, that will appear higher in energy and corresponding with larger deformations.

The concept of QPT refers to the sudden change of the atomic nucleus ground state structure as a function of a control parameter. Such control parameter can be, e.g., the neutron number and, therefore, a QPT can appear in an isotopic chain where the ground state deformation varies in an abrupt way when passing from an isotope to its neighbor. Taking into account that the two-neutron separation energy $\left(\mathrm{S}_{2 n}\right)$ is somehow proportional to the derivative of the energy, a first-order QPT will involve a discontinu- 
ity in $\mathrm{S}_{2 n}$ while a second-order one a discontinuity in the derivative of $S_{2 n}$. In this contribution we are mainly interested in the case of first-order QPT because it allows the coexistence of two phases around the phase transition region.

An ideal framework to deal with QPTs are the algebraic models, such as the interacting boson model (IBM) [3]. In such a framework a QPT can be modeled in terms of a Hamiltonian that is a combination of two given symmetries of the system, combined through a control parameter, $H=x H_{\text {sym } 1}+(1-x) H_{\text {sym2 }}$. The onset of a QPT is denoted by the existence of a critical value of the control parameter, $\mathrm{x}_{c}$, for which the structure of the system passes from one phase with symmetry 1 to another phase with symmetry 2. a)

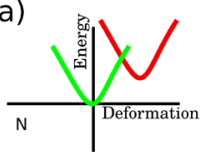

b)

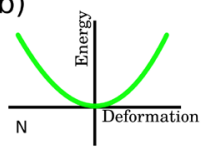

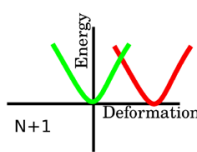

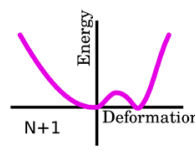

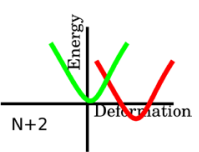

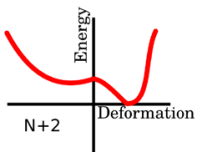

Figure 1. Schematic representation (both scales in arbitrary units) of the evolution of the energy surface for shape coexistence (a) and QPT (b) cases, as a function of the neutron number, $N$.

Are shape coexistence and QPT related in any way? On one hand, both phenomena involve a rapid change in the structure of a certain set of states, either ground or excited states. On the other hand, in both cases, at the meanfield level, several minima coexist. In a pictorial way, in Fig. 1 we depict the evolution of two minima as a function of the neutron number. In panel a) we treat the shape coexistence situation where the relative position of the regular (green) and intruder (red) minima changes as a function of the neutron number. In this case, we assume that intruder and regular minima become degenerated and even that the intruder describes the ground state of the system, although this situation is not general and in many cases the intruder band never becomes the ground state. In panel b) we consider the QPT case, for which in the left most panel only one spherical minimum exists, in the central one an energy surface with two minima develops and finally in the right most panel the spherical minimum disappears and only the deformed one remains. The case where the two minima coexist leads to the existence of a QPT. Obviously both situations present clear similarities, namely, around the region of interest two minima coexist and the value of the deformation changes abruptly in the central panels. However, at the quantum level one expects to observe clear differences. For example, the height of the barrier between both configurations should be a clear source of differences because the states of the bands with corresponding differ- ent wells will effectively interact for low barriers while remain unperturbed for high ones. In the figure, we depicted the case of a high barrier with no interacting configurations for the case of shape coexistence, while a low one for QPT. Note that other situations can also exist.

\section{$3 \mathrm{Zr}, \mathrm{Pt}$, and $\mathrm{Hg}$}

The $\mathrm{Pb}$ region is, probably, the best example of shape coexistence along the mass table and, indeed, in $\mathrm{Pb}$ up to three different configurations have been identified. In the $\mathrm{Hg}$ and $\mathrm{Pt}$ nuclei, which are the nuclei we are interested in, two kind of configurations show up with a clear presence of low lying $0^{+}$states, combined with a parabolic shape in the excitation energy systematics in $\mathrm{Hg}$, whereas not so obvious for Pt. On the other hand, $\mathrm{Zr}$ is known to exhibit the faster change in deformation ever observed, with ${ }^{98} \mathrm{Zr}$ almost spherical and ${ }^{100} \mathrm{Zr}$ a very well deformed nucleus.
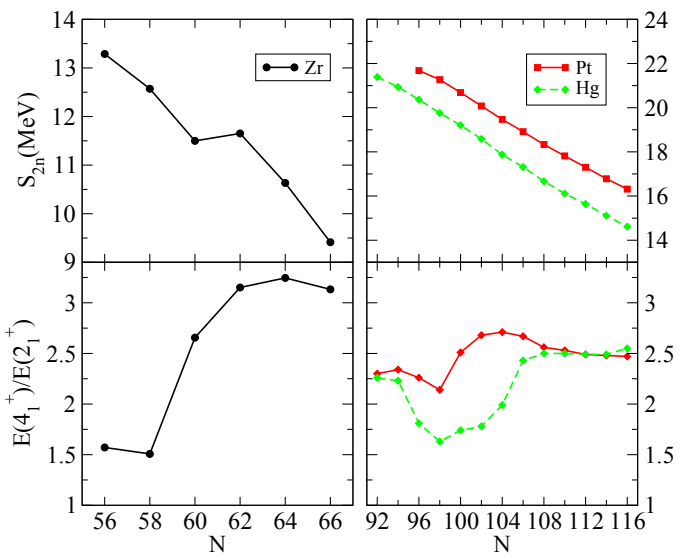

Figure 2. $\mathrm{S}_{2 n}$ and $E\left(4_{1}^{+}\right) / E\left(2_{1}^{+}\right)$as a function of the neutron number, $N$, for $\mathrm{Zr}$, Pt and $\mathrm{Hg}$.

From the point of view of a QPT, there are two key observables that can be used as indicators of the onset of a QPT. The first one is the $S_{2 n}$ that will experience a discontinuity at the first-order transition point, and the second one is the ratio between the excitation energies of the states $2_{1}^{+}$and $4_{1}^{+}, E\left(4_{1}^{+}\right) / E\left(2_{1}^{+}\right)$, which is related with the deformation or, in other words, with the value of the order parameter of the QPT. In Fig. 2 we present the systematics of $\mathrm{S}_{2 n}$ and $E\left(4_{1}^{+}\right) / E\left(2_{1}^{+}\right)$, as well, for $\mathrm{Zr}$ and $\mathrm{Pt}$ and $\mathrm{Hg}$. Regarding the value of $S_{2 n}$, one notices a clear discontinuity in $\mathrm{Zr}$, but a fully linear behavior in $\mathrm{Hg}$ and $\mathrm{Pt}$. On the other hand, $E\left(4_{1}^{+}\right) / E\left(2_{1}^{+}\right)$shows for $\mathrm{Zr}$ the typical rapid change that is observed in a QPT, passing from a value close to 2 (even 1.5 for ${ }^{96-98} \mathrm{Zr}$ ) to another close to 3.3 . However, in $\mathrm{Pt}$ and $\mathrm{Hg}$ the interpretation of the systematics is not so obvious. While in Pt it partially resembles what is observed in a QPT, in $\mathrm{Hg}$ there is a noticeable dropping of the ratio around mid-shell. In the latter two cases the systematics can be understood in terms of the coexistence of two configurations that cross or approach very closely. 
To shed some more light on the problem, in Fig. 3 we show results corresponding to IBM calculations that were presented elsewhere ([4] for $\mathrm{Pt},[5]$ for $\mathrm{Hg}$ and [6] for $\mathrm{Zr}$ ). In particular, the results correspond to excitation energies of selected states, both regular and intruder, for which the interaction term between intruder and regular sectors of the Hamiltonian has been switched off. In these three cases one observes a rather flat energy systematics for the intruder states while the conspicuous parabolic behaviour for the intruder ones centered around the mid-shell ( $A=106,182$, and 184 for $\mathrm{Zr}, \mathrm{Pt}$, and $\mathrm{Hg}$, respectively). However, several distinct features are worth to be mentioned. First, in $\mathrm{Zr}$ and $\mathrm{Pt}$ nuclei, a intruder state becomes the ground state around the mid-shell, while it never happens for $\mathrm{Hg}$. That means that the structure of the ground state changes abruptly around the mid-shell in $\mathrm{Zr}$ and $\mathrm{Pt}$, but not in $\mathrm{Hg}$. In $\mathrm{Hg}$ a change in the structure is observed in the $2^{+}$states. Note that the more realistic calculation involves a certain degree of mixing between the regular and the intruder states, which is rather small in $\mathrm{Zr}$ [6] and in $\mathrm{Hg}$ [5], but much larger in $\mathrm{Pt}$ [4], which means that the observed behavior in $\mathrm{Pt}$ is smoothed out in the more realistic calculation, but remains essentially the same in $\mathrm{Zr}$ and $\mathrm{Hg}$.

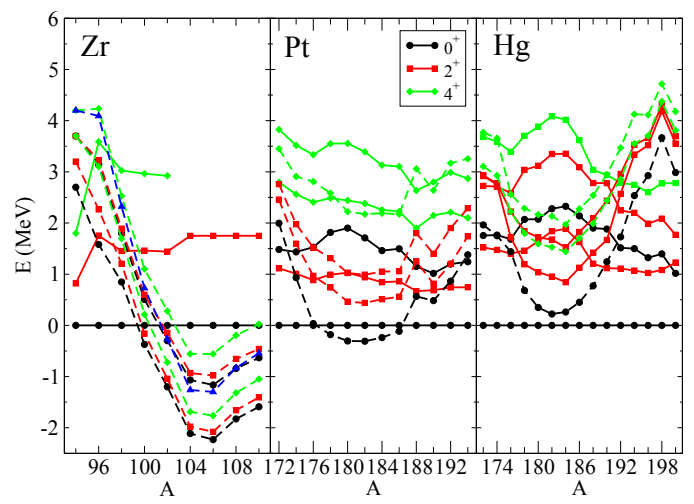

Figure 3. Theoretical IBM excitation energies for selected states in $\mathrm{Zr}, \mathrm{Pt}$, and $\mathrm{Hg}$ and Hamiltonians without interactions between the regular and the intruder sectors, as a function of $A$. Full lines stand for regular states and dashed ones for intruder ones.

The above features have direct consequences in the systematics of $\mathrm{S}_{2 n}$ and $E\left(4_{1}^{+}\right) / E\left(2_{1}^{+}\right)$. In the case of $\mathrm{Zr}$, as observed in Fig. 2 can be easily understood in terms of the crossing of configurations depicted in Fig. 3. Indeed, the change in the nature of the ground state produces a sudden change in the slope of the energy systematics and therefore a discontinuity in the value of $\mathrm{S}_{2 n}$. On the other hand, because the crossing is so fast, not only the ground state presents an intruder character, but also the rest of lowlying members of the yrast band involved in the energy ratio $E\left(4_{1}^{+}\right) / E\left(2_{1}^{+}\right)$, which makes that for $A<100$ all the states present a vibrational character, while for $A \geq 100$ a rotational one appears. On the other hand, in the case of $\mathrm{Hg}$, because the ground state exhibits a regular character and, moreover, there is no interaction between intruder and regular sector, the linear tendency of $\mathrm{S}_{2 n}$ even at the midshell can be understood. Besides, the crossing between $2_{1}^{+}$ and $4_{1}^{+}$regular and intruder states can explain the drop of the ratio $E\left(4_{1}^{+}\right) / E\left(2_{1}^{+}\right)$around the mid-shell. Finally, to explain the systematics of Pt in Fig. 2 is a challenge. First, the crossing of regular and intruder $0^{+}$energies would suggest a discontinuity in $\mathrm{S}_{2 n}$ as the observed one in $\mathrm{Zr}$. However experimental values show a fully linear systematics. The reason for such a behaviour is the strong mixing between the regular and the intruder sectors (see [4]). On the other hand, the energy ratio $E\left(4_{1}^{+}\right) / E\left(2_{1}^{+}\right)$seems to present the precursors of a QPT, but they are hindered. Most probably, this too is due to the strong mixing between the regular and intruder states. Note that according to [7] the value of the deformation, both in the IBM and in the HFB calculations, changes as it should be in a QPT. However the strong mixing somehow hides its effect in both the $\mathrm{S}_{2 n}$ and $E\left(4_{1}^{+}\right) / E\left(2_{1}^{+}\right)$observables.

\section{Conclusions}

We have analyzed the interplay between shape coexistence and QPT in three chain of isotopes, namely $\mathrm{Zr}$, Pt, and $\mathrm{Hg}$, that seem to be the most promising candidates to disentangle the relationship between both phenomena. In the case of $\mathrm{Zr}$, the QPT that appears around $A=100$ is easily explained in terms of the existence of two families of states that cross at the phase transition point. In $\mathrm{Hg}$, because the ground state never becomes of intruder character, no indications of QPT are shown, neither for $S_{2 n}$ not for $E\left(4_{1}^{+}\right) / E\left(2_{1}^{+}\right)$. Finally, in $\mathrm{Pt}$, the crossing of regular and intruder configurations could induce a QPT, but it is not observed in $\mathrm{S}_{2 n}$ and only partially in $E\left(4_{1}^{+}\right) / E\left(2_{1}^{+}\right)$. The reason for that is most probably the strong mixing between regular and intruder configurations. Therefore, we have found a mechanism for inducing a QPT from shape coexistence, though probably is not the only one.

Financial support from the Interuniversity Attraction Poles Program of the Belgian State-Federal Office for Scientific, Technical and Cultural Affairs (IAP Grant No. P7/12) and the Spanish MINECO and FEDER under Project No. FIS2014-53448-C2-2-P are acknowledged.

\section{References}

[1] P. Cejnar, J. Jolie, and R.F. Casten, Rev. Mod. Phys. 82, 2155 (2010).

[2] K. Heyde and J.L. Wood, Rev. Mod. Phys. 83, 1467 (2011).

[3] F. Iachello and A. Arima, The Interacting Boson Model, Cambridge University Press (1987).

[4] J.E. García-Ramos and K. Heyde, Nucl. Phys. A 825, 39 (2009); J.E. García-Ramos, V. Hellemans, and K. Heyde, Phys. Rev. C 84, 014331 (2011).

[5] J.E. García-Ramos, and K. Heyde, Phys. Rev. C 89, 014306 (2014).

[6] J.E. García-Ramos and K. Heyde, work in progress.

[7] J.E. García-Ramos, K. Heyde, L.M. Robledo, and R. Rodriguez-Guzmán, Phys. Rev. C 89, 034313 (2014). 